\title{
VARIAÇÃO DIURNO-NOTURNA DO POTENCIAL MÁTRICO E GRADIENTE DE POTENCIAL TOTAL DA ÁGUA NO SOLO ${ }^{(1)}$
}

\author{
Alexsandro dos Santos Brito ${ }^{(2)}$, Paulo Leonel Libardi ${ }^{(3)}$, Jaedson Cláudio Anunciato \\ Mota $^{(4)}$ \& Vilson Antonio Klein ${ }^{(5)}$
}

\begin{abstract}
RESUMO
O potencial mátrico em solos não saturados é um importante componente do potencial total e, portanto, deve ser medido da melhor forma possível. Este trabalho teve por objetivo avaliar a influência da temperatura do solo sobre o potencial mátrico e o gradiente de potencial total, durante a redistribuição da água num Latossolo Vermelho-Amarelo, em Piracicaba, SP, a partir de dados coletados por tensiometria. Em uma parcela circular de $3 \mathrm{~m}$ de diâmetro, foram instalados 10 tensiômetros com transdutor de tensão, modelo SWT3 da Delta-T, nas profundidades de 0,$10 ; 0,15 ; 0,20 ; 0,25 ; 0,30 ; 0,35 ; 0,40 ; 0,45 ; 0,50 ;$ e $0,55 \mathrm{~m}$, com os quais foram obtidos os potenciais mátricos para essas profundidades e o gradiente de potencial total, $(\Delta \phi \mathrm{m} / \Delta \mathrm{z})+1$, para a profundidade de $0,25 \mathrm{~m}, \operatorname{com} \Delta \mathrm{z}$ de 0,$1 ; 0,2$; e $0,3 \mathrm{~m}$, utilizando os tensiômetros instalados nas profundidades de 0,20 e 0,$30 ; 0,15$ e 0,35; e 0,10 e 0,40 m para $\Delta \phi \mathrm{m}$, respectivamente. Na mesma parcela, foram também instalados termopares tipo $\mathrm{T}$ na superfície e nas profundidades 0,$025 ; 0,050 ;$ e 0,075 $\mathrm{m}$ de solo. Ambas as leituras dos tensiômetros e termopares foram armazenadas em um Datalogger, Modelo DL2, da Delta-T, a idênticos intervalos de tempo. As avaliações detectaram oscilações na leitura do potencial mátrico da água no solo ao longo do dia e nos efeitos diferenciados nos tensiômetros instalados em profundidades diferentes, ocasionando oscilações também no gradiente de potencial total da água no solo. Os melhores horários para a realização das leituras dos tensiômetros são nas primeiras sete horas do dia ou após às $18 \mathrm{~h}$.
\end{abstract}

Termos de indexação: temperatura do solo, transdutor de tensão, tensiômetro.

(1) Recebido para publicação em 16 de agosto de 2012 e aprovado em 25 de outubro de 2013.

${ }^{(2)}$ Professor Efetivo, Instituto Federal de Educação, Ciência e Tecnologia Baiano, Campus Guanambi. Distrito de Ceraíma, Zona Rural. CEP 46430-000 Guanabi (BA). E-mail: alexsandrobrt@gmail.com

(3) Professor Titular, Departamento de Engenharia de Biossistemas, ESALQ/USP. Av. Pádua Dias, 11, São Dimas. Caixa Postal 9. CEP 13418-900 Piracicaba (SP). Bolsista de Produtividade do CNPq. E-mail: pllibardi@usp.br

(4) Professor Adjunto, Universidade Federal do Ceará. R. Paulino Nogueira, s/n, Benfica. CEP 60020-270 Fortaleza (CE). E-mail: jaedsonmota@yahoo.com.br

(5) Professor, Faculdade de Agronomia e Medicina Veterinária, Universidade de Passo Fundo. BR 285, Bairro São José. CEP 99052-900 Passo Fundo (RS). E-mail: vklein@upf.br 


\title{
SUMMARY: DIURNAL-NOCTURNAL FLUCTUATION OF SOIL WATER MATRIC POTENTIAL AND SOIL WATER TOTAL POTENTIAL GRADIENT
}

\begin{abstract}
As the water matric potential is the most important component of the water total potential in unsaturated soils, it must be measured accurately. The aim of this study was to evaluate the effect of soil temperature on water matric potential and water total potential gradient over the period of water redistribution in a Latossolo Vermelho-Amarelo (Oxisol/Hapludox) located in the municipality of Piracicaba, SP, Brazil. In a circular plot of 3 m diameter, 10 tensiometers with pressure transducers (model SWT3 from Delta-T Devices) were set up at depths of 0.10, $0.15,0.20,0.25,0.30,0.35,0.40,0.45,0.50$ and $0.55 \mathrm{~m}$ in order to determine water matric potential, $\Phi m$, at those depths and to calculate the water total potential gradient, $(\Delta \Phi m /$ $\Delta z)+1$, at the depth of $0.25 \mathrm{~m}$ with $\Delta z$ of $0.10 ; 0.20$ and $0.30 \mathrm{~m}$ (using tensiometers set up at 0.20 and 0.30; 0.15 and 0.35 , and 0.10 and $0.40 \mathrm{~m}$ for $\Delta \Phi m$, respectively). In the same plot, thermocouples (type T) were set up at the soil surface and at the depths of 0.025, 0.05 and 0.075 $m$. Tensiometer and thermocouple readings were made simultaneously and stored in a Datalogger (model DL2, Delta-T Devices). Measured values of soil water matric potential exhibit fluctuations throughout the day and differential effects for tensiometers at different depths, also causing fluctuations in the soil water total potential gradient. The best time period for performing tensiometer readings is in the first seven hours of the day or after $6 \mathrm{pm}$.
\end{abstract}

Index terms: soil temperature, pressure transducer, tensiometer.

\section{INTRODUÇÃO}

O manejo adequado de um sistema de produção agrícola exige conhecimento aprofundado sobre uma série de fatores e da relação entre eles. Nesse contexto, para a adoção de um manejo racional da água no solo, do solo e da cultura, é condição indispensável o conhecimento a respeito do componente água no solo, como fator principal de produção agrícola. A dinâmica da água no solo é regida, fundamentalmente, pelo seu estado energético em diferentes camadas do perfil do solo, daí ser bastante comum encontrar na literatura as expressões "potencial total da água", "gradiente de potencial total", as quais expressam o potencial hídrico do solo em termos de transporte.

Para as condições de solo não saturado, há um componente importante para a quantificação do estado energético da água, oriundo da força com que a água é retida pela matriz sólida, denominado de "potencial mátrico". Esse potencial, somado ao "potencial gravitacional", medido em relação a um plano referencial, configura à água um estado energético chamado de "potencial total". É a diferença de potencial total da água no solo entre dois pontos que faz com que haja movimento da água do ponto de maior para o de menor potencial total. Essa diferença de potencial, dividida pela distância entre os pontos, é denominada de "gradiente de potencial total". Portanto, é de grande valia o entendimento de como se comporta o potencial mátrico da água no solo para, a partir dele, manejar o sistema solo-água-planta da melhor maneira possível (Wang et al., 2007).

O tensiômetro, idealizado em torno de 1908 por Burton E. Livingston (Or, 2001), é um dos instrumentos mais difundidos para a medida do potencial mátrico da água no solo. É bastante simples e consiste, basicamente, de uma cápsula porosa aderida a um tubo com um manômetro. Vários modelos têm sido propostos para melhorar a versatilidade, principalmente quanto à faixa de operação (Whalley et al., 2007; Bakker et al., 2007; van der Ploeg et al., 2008). Os transdutores de tensão têm sido apresentados na literatura como uma alternativa ao tensiômetro convencional (Marthaler et al., 1983; Nyran \& Drennon, 1990; Azzoz \& Arshad, 1994; Hubbell \& Sisson, 1998; Klein et al., 2004; Moraes et al., 2006). Esses autores destacaram a funcionalidade do uso de tensiômetros com transdutores de tensão acoplados a sistemas eletrônicos de aquisição de dados em pesquisas de campo. Para Klein (2001), o inconveniente desses modelos é que ainda são relativamente caros, quando comparados ao modelo com manômetro de mercúrio, por envolverem complexos sistemas eletrônicos.

A variação diurna do potencial mátrico da água no solo, medida pelo tensiômetro, não está completamente relacionada às mudanças do conteúdo de água no solo, ou seja, existe a influência de outros fatores (Haise \& Kelley, 1950). Considerando essa hipótese, é necessário conhecer os fatores que interferem nessa medida e o comportamento dos tensiômetros em relação a eles; por exemplo, o tipo de instrumento para a medida do potencial mátrico, o tempo de equilíbrio da tensão da água no solo com a tensão no interior do instrumento e o horário de leitura, em razão da influência dos fatores climáticos, são muito importantes para a correta tomada dos dados (Brito et al., 2009).

A propósito, os tensiômetros que operam próximos à superfície do solo são influenciados pela temperatura (Warrick et al., 1998), tornando importante conhecer o comportamento desses equipamentos em relação a 
essa variável especificamente. Segundo os autores, as flutuações nas leituras dos tensiômetros, por causa das variações de temperatura na superfície do solo, tornam complicada a medida de potencial mátrico da água no solo. Quanto aos gradientes hidráulicos, a determinação é mais difícil quando dois ou mais tensiômetros são influenciados diferentemente por essas variações.

A influência da temperatura nos parâmetros que determinam o potencial mátrico, como é o caso da tensão superficial $(\sigma)$ e do ângulo de contato $(\gamma)$, é insuficiente para gerar modificações significativas, uma vez que para a tensão superficial há redução de apenas $4,5 \%$ entre as temperaturas de 20 e $40{ }^{\circ} \mathrm{C}$ e aumento no ângulo de contato de 1,0 para $8,5^{\circ}$, com o aumento da temperatura de 5,0 para $38^{\circ} \mathrm{C}$ (Dexter et al., 2010). Entretanto, a temperatura pode causar modificações indiretas no ângulo de contato, por promover a dissolução de substâncias (efeito tixotrópico, principalmente em substâncias orgânicas) e essas reduzirem o ângulo de contato. $\mathrm{O}$ aumento da pressão do ar aprisionado nos poros do solo também seria um fator; essa pressão é transmitida para a água contida nos poros, reduzindo o potencial mátrico da água no solo (Dexter et al., 2010).

Desse modo, a pesquisa teve o objetivo de verificar a influência da temperatura do solo no potencial mátrico e no gradiente de potencial total, durante a redistribuição da água em um Latossolo, em Piracicaba, SP, a partir de dados coletados com tensiômetros com transdutor de tensão.

\section{MATERIAL E MÉTODOS}

O experimento foi conduzido em uma área experimental do campus da Escola Superior de Agricultura "Luiz de Queiroz", em Piracicaba, SP, por um período de 10 dias. O solo é classificado como Latossolo Vermelho-Amarelo distrófico e o clima é Cwa, pela classificação de Köppen.

Foram instalados 10 tensiômetros com transdutor de pressão, instalado na extremidade superior do tensiômetro, modelo SWT3-AT (Delta-T), nas profundidades de 0,$10 ; 0,15 ; 0,20 ; 0,25 ; 0,30 ; 0,35$; 0,$40 ; 0,45 ; 0,50 ;$ e $0,55 \mathrm{~m}$. Esse modelo de transdutor de pressão tem faixa de temperatura para funcionamento de -20 a $50{ }^{\circ} \mathrm{C}$ (desvio de $0,5 \%$ ). Por problemas de funcionamento, o tensiômetro a $0,25 \mathrm{~m}$ de profundidade teve que ser desconsiderado da avaliação. Os tensiômetros foram confeccionados com tubos de PVC rígido, com diâmetros externo e interno medindo 0,021 e 0,016 m, respectivamente, e comprimento correspondente a cada profundidade de instalação, somado mais $0,3 \mathrm{~m}$.

A instalação dos instrumentos foi feita em uma parcela circular de $3 \mathrm{~m}$ de diâmetro, delimitada por uma chapa metálica galvanizada ( $2 \mathrm{~mm}$ de espessura) até a profundidade de $1 \mathrm{~m}$, objetivando elevar o conteúdo de água no solo ao máximo. Foi deixado 0,20 m da chapa metálica acima da superfície do solo para manter uma lâmina de água de 0,15 a 0,20 m e facilitar o processo de saturação do solo.

Após a instalação, a área foi inundada de modo a garantir a saturação do perfil até a profundidade de $0,6 \mathrm{~m}$. Após a operação de saturação, a parcela foi coberta com lona plástica para evitar qualquer fluxo de água através da superfície, seja por evaporação ou infiltração. Sobre a lona plástica, colocou-se palhada de capim braquiária para minimizar o aquecimento nas horas mais quentes do dia, em virtude da incidência direta da radiação solar. A partir do momento de colocação dessa lona, foram registradas leituras dos tensiômetros a cada 5 min e armazenadas em um Datalogger, Modelo DL2 (Delta-T). O Datalogger foi programado para converter os dados de leitura, em $\mathrm{mV}$, para potencial mátrico, utilizando equações adaptadas para cada profundidade de instalação.

Paralelamente, registraram-se as temperaturas na superfície do solo e nas profundidades de 0,$025 ; 0,050$; e $0,075 \mathrm{~m}$, por meio de termopares tipo T, com armazenamento dos dados no mesmo Datalogger e nos mesmos intervalos de tempo.

Com os potencias mátricos, foram calculados os potenciais totais para cada profundidade, a partir da equação 1:

$$
\phi_{t}=\phi_{m}+\phi_{g}
$$

em que $\phi_{t}$ é o potencial total (m); $\phi_{m}$, o potencial mátrico (m); e $\phi_{g}$, o potencial gravitacional (m), considerado como sendo a distância do centro da cápsula do tensiômetro em relação ao nível do solo.

O gradiente de potencial total da água no solo foi calculado com $\Delta z$ de $0,10 \mathrm{~m}$ (tensiômetros nas profundidades de 0,20 e $0,30 \mathrm{~m}$ ); de $0,20 \mathrm{~m}$ (tensiômetros nas profundidades de 0,15 e 0,35 m) e de $0,30 \mathrm{~m}$ (tensiômetros nas profundidades de 0,10 e 0,40 m), em função dos 24 períodos do dia.

$$
\text { Grad } \phi_{t}=\left[\frac{\phi_{t_{(C)}}-\phi_{t_{(B)}}}{\Delta z}\right]
$$

em que $\operatorname{Grad} \phi_{t}$ é o gradiente de potencial total da água (m); $\phi_{t(\mathrm{~B})}$, o potencial total no ponto de baixo (m); $\phi_{t(\mathrm{C}),}$ o potencial total no ponto de cima (m); e $\Delta z$, a distância, em $\mathrm{m}$, entre os pontos.

Para entender o comportamento do potencial mátrico da água no solo ao longo do dia (leituras feitas do segundo, 10/02/2007, ao $11^{\circ}$ dia de redistribuição da água no solo, 19/02/2007), dividiu-se o dia em 24 períodos de $1 \mathrm{~h}$. Esse escalonamento foi adotado para identificar influências da temperatura do ar e do solo sobre as variáveis em análise. Para tanto, realizou-se a mesma divisão para os dados de temperatura.

$\mathrm{O}$ delineamento experimental foi o inteiramente aleatorizado, com 24 tratamentos ( 24 períodos de $1 \mathrm{~h}$ ) 
e 10 repetições (10 dias de avaliação). Os dados foram submetidos à análise de variância, pelo teste $\mathrm{F}$, e as médias, que representam as medidas tomadas em cada período de $1 \mathrm{~h}$, nas $24 \mathrm{~h}$ do dia, foram comparadas pelo teste de Tukey a $5 \%$.

\section{RESULTADOS E DISCUSSÃO}

Os valores de potencial mátrico $(-\mathrm{kPa})$ da água no solo em cada profundidade ao longo dos 10 dias de avaliação (Figura 1a) diminuíram lentamente com o decorrer do tempo. Observou-se que à medida que o tempo decorreu, menores são as variações do potencial mátrico em cada profundidade, evidenciando a retenção de água contra o campo gravitacional não apenas pela menor $\partial \mathrm{q} / \partial \mathrm{t}$, mas também pelo conteúdo de água mantido mesmo depois de cerca de $300 \mathrm{~h}$. Os picos observados no potencial mátrico ao longo do tempo nas profundidades avaliadas (Figura 1a) seguiram a mesma tendência da temperatura do solo (Figura 1b). Analisando os 24 períodos estudados (Quadro 1), os valores de potencial mátrico diminuíram do $9^{\circ}$ até o $16^{\circ}$ período (entre 15 e $16 \mathrm{~h}$ ) e aumentaram do $16^{\circ}$ ao $20^{\circ}$ período, mantendo-se constante nos demais períodos, nas nove profundidades avaliadas.

O potencial mátrico da água no solo medido em todas as profundidades seguiu uma mesma tendência (Quadro 1), ou seja, teve seu valor diminuído, principalmente no período de maior temperatura do ar, determinado na superfície do solo (sob cobertura plástica), e do solo $(0,025 ; 0,050$ e $0,075 \mathrm{~m}$ de profundidade). Essa tendência também foi verificada por Buchter et al. (1999), os quais observaram diminuição no potencial mátrico a partir das $10 \mathrm{~h}$ e aumento a partir das $18 \mathrm{~h}$, diferindo deste trabalho apenas nos períodos de maior influência da temperatura e da radiação solar, em razão da posição geográfica de cada estudo. Coelho \& Teixeira (2004) verificaram erro de aproximadamente $-1,5 \mathrm{kPa}$ no potencial mátrico da água no solo medido ao meio-dia e que o erro causado pela elevação da temperatura é maior para potenciais mátricos menores.

Neste estudo, a redistribuição da água no solo é o processo que está conduzindo a uma diminuição do potencial mátrico com o tempo. Entretanto, o potencial mátrico retorna a um valor próximo ao inicial, ainda em períodos diurnos (Quadro 1), indicando uma correlação clara com a temperatura, como ilustrado pelas regressões lineares entre os valores de temperatura do solo (média das temperaturas medidas nas profundidades de 0,$025 ; 0,05 ;$ e $0,075 \mathrm{~m}$ ) e potencial mátrico $(-\mathrm{kPa})$, nas profundidades avaliadas (Figura 2). Esse comportamento pode estar relacionado com o efeito tixotrópico e o aumento da pressão do ar aprisionado nos poros (Dexter et al., 2010). Em estudos de campo, é muito difícil conseguir uma perfeita saturação do solo em razão da conformação e tortuosidade dos poros, ficando sempre o ar aprisionado que pode influenciar a medida do potencial mátrico, mesmo em conteúdos de água próximos da saturação, em virtude da variação da pressão desse ar aprisionado na matriz do solo, promovida pela oscilação térmica. Com a saída de água do volume de solo considerado, os dois efeitos avaliados por Dexter et al. (2010) podem se tornar mais intensos, uma vez que a variação térmica tende aumentar.

Nas profundidades de 0,2 e 0,4 m, por exemplo, o coeficiente de determinação foi elevado, 0,84 e 0,80 , respectivamente, mesmo a medida da temperatura não sendo pontual como foi a do potencial mátrico, indicando que é possível alcançar melhores ajustes caso a temperatura do solo seja medida na mesma profundidade de instalação da cápsula porosa. Bakker et al. (2007) também verificaram picos nas medidas de potencial mátrico quando ocorreram mudanças abruptas de temperatura, ao avaliarem tensiômetros construídos à base de um polímero que permite ampliar a faixa de tensão de funcionamento desse em relação aos convencionais.

A influência da temperatura na medida do potencial mátrico está relacionada também com o
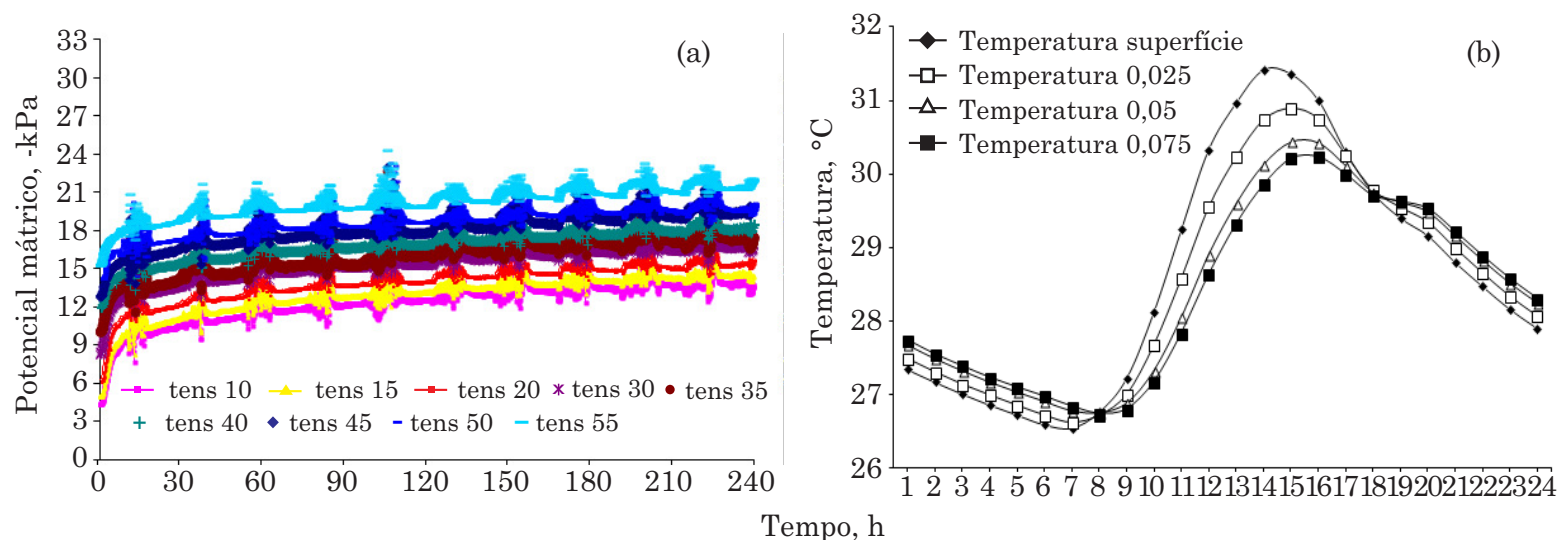

Figura 1. Potencial mátrico da água no solo (a) e temperatura média por período (b), durante os 10 dias de monitoramento. 
Quadro 1. Potencial mátrico e erros-padrão máximo e mínimo da média (EPM) em função dos 24 períodos, nas profundidades avaliadas

\begin{tabular}{|c|c|c|c|c|c|c|c|c|c|}
\hline \multirow{3}{*}{ Período } & \multicolumn{9}{|c|}{ Potencial mátrico } \\
\hline & \multicolumn{9}{|c|}{ Profundidade (m) } \\
\hline & 0,10 & 0,15 & 0,20 & 0,30 & 0,35 & 0,40 & 0,45 & 0,50 & 0,55 \\
\hline & & & & & $-\mathrm{kPa}$ & & & & \\
\hline 1 & $12,50 \mathrm{IJ}$ & $13,18 \mathrm{~L}$ & $14,06 \mathrm{I}$ & $15,50 \mathrm{H}$ & $16,03 \mathrm{~J}$ & $17,02 \mathrm{G}$ & $18,08 \mathrm{H}$ & $18,69 \mathrm{IJ}$ & $20,18 \mathrm{G}$ \\
\hline 2 & $12,52 \mathrm{IJ}$ & $13,20 \mathrm{~L}$ & $14,06 \mathrm{I}$ & $15,50 \mathrm{H}$ & $16,02 \mathrm{~J}$ & $17,04 \mathrm{G}$ & $18,08 \mathrm{H}$ & $18,68 \mathrm{IJ}$ & $20,17 \mathrm{G}$ \\
\hline 3 & $12,51 \mathrm{IJ}$ & $13,21 \mathrm{~L}$ & $14,08 \mathrm{I}$ & $15,50 \mathrm{H}$ & $16,02 \mathrm{~J}$ & $17,05 \mathrm{G}$ & $18,09 \mathrm{H}$ & $18,67 \mathrm{IJ}$ & $20,18 \mathrm{G}$ \\
\hline 4 & $12,55 \mathrm{IJ}$ & $13,25 \mathrm{KL}$ & $14,11 \mathrm{I}$ & $15,54 \mathrm{H}$ & $16,07 \mathrm{IJ}$ & $17,08 \mathrm{G}$ & $18,12 \mathrm{H}$ & $18,70 \mathrm{IJ}$ & $20,20 \mathrm{G}$ \\
\hline 5 & $12,57 \mathrm{IJ}$ & $13,27 \mathrm{KL}$ & $14,12 \mathrm{I}$ & $15,55 \mathrm{H}$ & $16,09 \mathrm{IJ}$ & $17,09 \mathrm{G}$ & $18,13 \mathrm{H}$ & $18,71 \mathrm{IJ}$ & $20,21 \mathrm{G}$ \\
\hline 6 & $12,59 \mathrm{IJ}$ & $13,29 \mathrm{KL}$ & $14,14 \mathrm{I}$ & $15,56 \mathrm{H}$ & 16,13 HIJ & $17,09 \mathrm{G}$ & $18,12 \mathrm{H}$ & $18,71 \mathrm{~J}$ & $20,20 \mathrm{G}$ \\
\hline 7 & $12,37 \mathrm{~J}$ & $13,18 \mathrm{~L}$ & $14,14 \mathrm{I}$ & $15,51 \mathrm{H}$ & $16,00 \mathrm{~J}$ & $17,07 \mathrm{G}$ & $18,17 \mathrm{H}$ & $18,62 \mathrm{HI}$ & $20,29 \mathrm{G}$ \\
\hline 8 & $12,56 \mathrm{IJ}$ & 13,41 LK & $14,44 \mathrm{H}$ & $15,80 \mathrm{G}$ & $16,29 \mathrm{GHI}$ & $17,34 \mathrm{~F}$ & $18,54 \mathrm{FG}$ & $18,86 \mathrm{FG}$ & $20,69 \mathrm{DE}$ \\
\hline 9 & $12,58 \mathrm{IJ}$ & $13,40 \mathrm{KJ}$ & $14,49 \mathrm{GH}$ & $15,80 \mathrm{G}$ & 16,33 FGH & $17,38 \mathrm{~F}$ & $18,57 \mathrm{FG}$ & $18,92 \mathrm{EFG}$ & $20,78 \mathrm{D}$ \\
\hline 10 & $12,72 \mathrm{HI}$ & $13,50 \mathrm{KIJ}$ & $14,62 \mathrm{FG}$ & $15,90 \mathrm{FG}$ & $16,49 \mathrm{EFG}$ & $17,48 \mathrm{EF}$ & $18,70 \mathrm{EF}$ & 19,06 FGH & $20,94 \mathrm{C}$ \\
\hline 11 & $12,70 \mathrm{HI}$ & $13,46 \mathrm{IJ}$ & $14,68 \mathrm{EF}$ & $15,93 \mathrm{FG}$ & $16,56 \mathrm{EF}$ & $17,54 \mathrm{E}$ & $18,74 \mathrm{DE}$ & $18,98 \mathrm{CD}$ & $21,03 \mathrm{BC}$ \\
\hline 12 & $13,06 \mathrm{FG}$ & $13,73 \mathrm{DEF}$ & $14,87 \mathrm{CD}$ & $16,16 \mathrm{DE}$ & $17,03 \mathrm{D}$ & $17,81 \mathrm{D}$ & $19,06 \mathrm{C}$ & $19,26 \mathrm{CDE}$ & $21,25 \mathrm{~A}$ \\
\hline 13 & $13,15 \mathrm{EF}$ & $13,72 \mathrm{EFG}$ & $14,86 \mathrm{CD}$ & $16,21 \mathrm{CD}$ & $17,13 \mathrm{CD}$ & $17,82 \mathrm{D}$ & $19,06 \mathrm{C}$ & $19,24 \mathrm{BC}$ & $21,23 \mathrm{~A}$ \\
\hline 14 & $13,31 \mathrm{DE}$ & $13,84 \mathrm{CDE}$ & $14,95 \mathrm{BC}$ & $16,35 \mathrm{BCD}$ & $17,36 \mathrm{C}$ & $17,98 \mathrm{BC}$ & $19,20 \mathrm{BC}$ & $19,38 \mathrm{~B}$ & $21,35 \mathrm{~A}$ \\
\hline 15 & $13,59 \mathrm{BC}$ & $14,01 \mathrm{BC}$ & $15,03 \mathrm{AB}$ & $16,54 \mathrm{~B}$ & $17,61 \mathrm{~B}$ & $18,04 \mathrm{AB}$ & $19,30 \mathrm{~B}$ & $19,51 \mathrm{~A}$ & $21,32 \mathrm{~A}$ \\
\hline 16 & $13,88 \mathrm{~A}$ & $14,22 \mathrm{~A}$ & $15,10 \mathrm{~A}$ & $16,77 \mathrm{~A}$ & $17,89 \mathrm{~A}$ & $18,19 \mathrm{~A}$ & $19,51 \mathrm{~A}$ & $19,83 \mathrm{~B}$ & $21,35 \mathrm{~A}$ \\
\hline 17 & $13,41 \mathrm{CD}$ & $13,91 \mathrm{BCD}$ & $14,77 \mathrm{DE}$ & $16,38 \mathrm{BC}$ & $17,27 \mathrm{CD}$ & $17,80 \mathrm{D}$ & $19,11 \mathrm{C}$ & 19,46 B & $21,04 \mathrm{BC}$ \\
\hline 18 & $13,43 \mathrm{CD}$ & $13,94 \mathrm{BC}$ & $14,84 \mathrm{CD}$ & $16,39 \mathrm{BC}$ & $17,21 \mathrm{CD}$ & $17,86 \mathrm{CD}$ & $19,05 \mathrm{C}$ & $19,53 \mathrm{~B}$ & $21,07 \mathrm{~B}$ \\
\hline 19 & $13,70 \mathrm{AB}$ & $14,02 \mathrm{~B}$ & $14,75 \mathrm{DE}$ & $16,49 \mathrm{~B}$ & $17,05 \mathrm{D}$ & $17,72 \mathrm{D}$ & $18,88 \mathrm{D}$ & $19,52 \mathrm{DEF}$ & $20,79 \mathrm{D}$ \\
\hline 20 & $13,10 \mathrm{EFG}$ & 13,66 FGH & $14,47 \mathrm{H}$ & $16,01 \mathrm{EF}$ & $16,65 \mathrm{E}$ & $17,42 \mathrm{EF}$ & $18,53 \mathrm{G}$ & $19,14 \mathrm{FGH}$ & $20,57 \mathrm{EF}$ \\
\hline 21 & 12,93 FGH & $13,58 \mathrm{GHI}$ & $14,42 \mathrm{H}$ & $15,90 \mathrm{FG}$ & $16,50 \mathrm{EFG}$ & $17,35 \mathrm{~F}$ & $18,45 \mathrm{G}$ & 19,04 FGH & $20,52 \mathrm{~F}$ \\
\hline 22 & 12,92 FGH & 13,57FGHI & $14,41 \mathrm{H}$ & $15,88 \mathrm{FG}$ & $16,48 \mathrm{EFG}$ & $17,35 \mathrm{~F}$ & $18,44 \mathrm{G}$ & 19,01 FGH & $20,51 \mathrm{~F}$ \\
\hline 23 & $12,88 \mathrm{GH}$ & $13,56 \mathrm{GHI}$ & $14,41 \mathrm{H}$ & $15,86 \mathrm{FG}$ & $16,43 \mathrm{EFG}$ & $17,34 \mathrm{~F}$ & $18,41 \mathrm{G}$ & $18,98 \mathrm{FGH}$ & $20,49 \mathrm{~F}$ \\
\hline 24 & $12,89 \mathrm{GH}$ & 13,57 FGHI & $14,41 \mathrm{H}$ & $15,86 \mathrm{FG}$ & $16,42 \mathrm{EFG}$ & $17,34 \mathrm{~F}$ & $18,42 \mathrm{G}$ & $18,96 \mathrm{FGH}$ & $20,49 \mathrm{~F}$ \\
\hline EPM Máx. & . 0,14 & 0,13 & 0,11 & 0,13 & 0,14 & 0,11 & 0,12 & 0,11 & 0,10 \\
\hline EPM Mín. & 0,08 & 0,09 & 0,08 & 0,08 & 0,08 & 0,07 & 0,08 & 0,06 & 0,07 \\
\hline
\end{tabular}

Médias seguidas da mesma letra nas colunas não diferem entre si pelo teste de Tukey a $5 \%$.

transdutor de pressão e com a flutuação da câmara de ar deixada entre a coluna de água no interior do tensiômetro e o transdutor de pressão. Por isso, é recomendável proteger o transdutor de pressão da radiação solar direta. Quanto à câmara de ar, formada em detrimento da saída de água do tensiômetro para o solo, recomenda-se manter a menor variação possível dentro de uma faixa, uma vez que há correlação positiva entre esse volume e o efeito da temperatura na medida do potencial mátrico (Butters \& Cardon, 1998). Como neste trabalho, a câmara de ar foi monitorada, restou a influência da temperatura no tensiômetro, inclusive no transdutor de pressão. No solo, a temperatura foi a fonte que ocasionou as variações do potencial mátrico, principalmente nos horários mais quentes do dia. A transmissão de energia térmica do ar atmosférico em conjunto com a do solo pode ocasionar alteração na tensão da água contida no interior do tensiômetro, como também na água retida pelo solo.
O gradiente de potencial total da água no solo também foi influenciado pela temperatura, apresentando comportamento bastante semelhante ao do potencial mátrico, com valores significativamente elevados no período das 11 às $19 \mathrm{~h}$, com pico em torno das $16 \mathrm{~h}$. O gradiente de potencial total é semelhante nas primeiras e últimas horas do dia (Figura 3a).

Os gradientes de potencial total medidos com $\Delta z$ de 0,30 e 0,20 m estão dentro da faixa de grandeza de outros estudos, como o de Pacheco et al. (1994), que utilizaram $\Delta z$ semelhantes a esses. A princípio, a medida do gradiente de potencial total com $\Delta z$ menor é mais real, uma vez que a proporcionalidade entre a diferença de potencial total e o $\Delta z$ não se mantém constante, principalmente em solos heterogêneos ou solos com camadas compactadas. Além disso, o efeito da temperatura parece ter sido diferente nos tensiômetros utilizados para essa medida, uma vez que o gradiente de potencial total apresentou picos semelhantes aos potenciais mátricos avaliados. Caso 
a influência fosse semelhante, o efeito ambiental seria anulado quando do cálculo do gradiente, o que não ocorreu.

Como o trabalho foi realizado em um Latossolo homogêneo, esperava-se que o gradiente de potencial total da água no solo fosse constante ao longo do dia, em razão das condições de contorno do experimento e das características físico-hídricas do solo. Entretanto, a temperatura parece ter influenciado de forma diferente os tensiômetros utilizados para essa medida, como também foi observado por Haise \& Kelley (1950) e Warrick et al. (1998). Provavelmente, o conteúdo

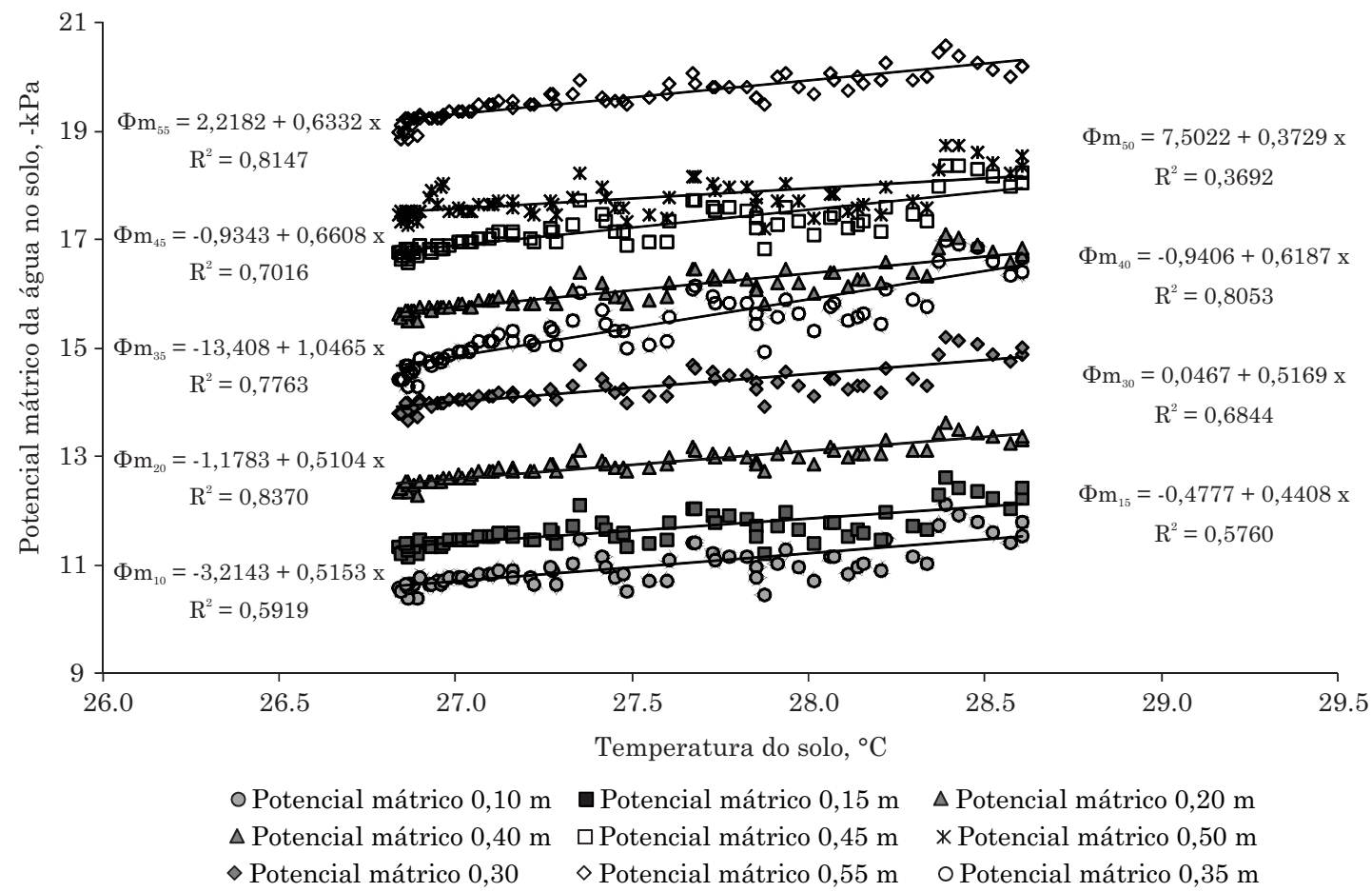

Figura 2. Regressões lineares do potencial mátrico $\left(\phi_{m}\right)$ em função da temperatura média do solo (C) nas profundidades avaliadas.
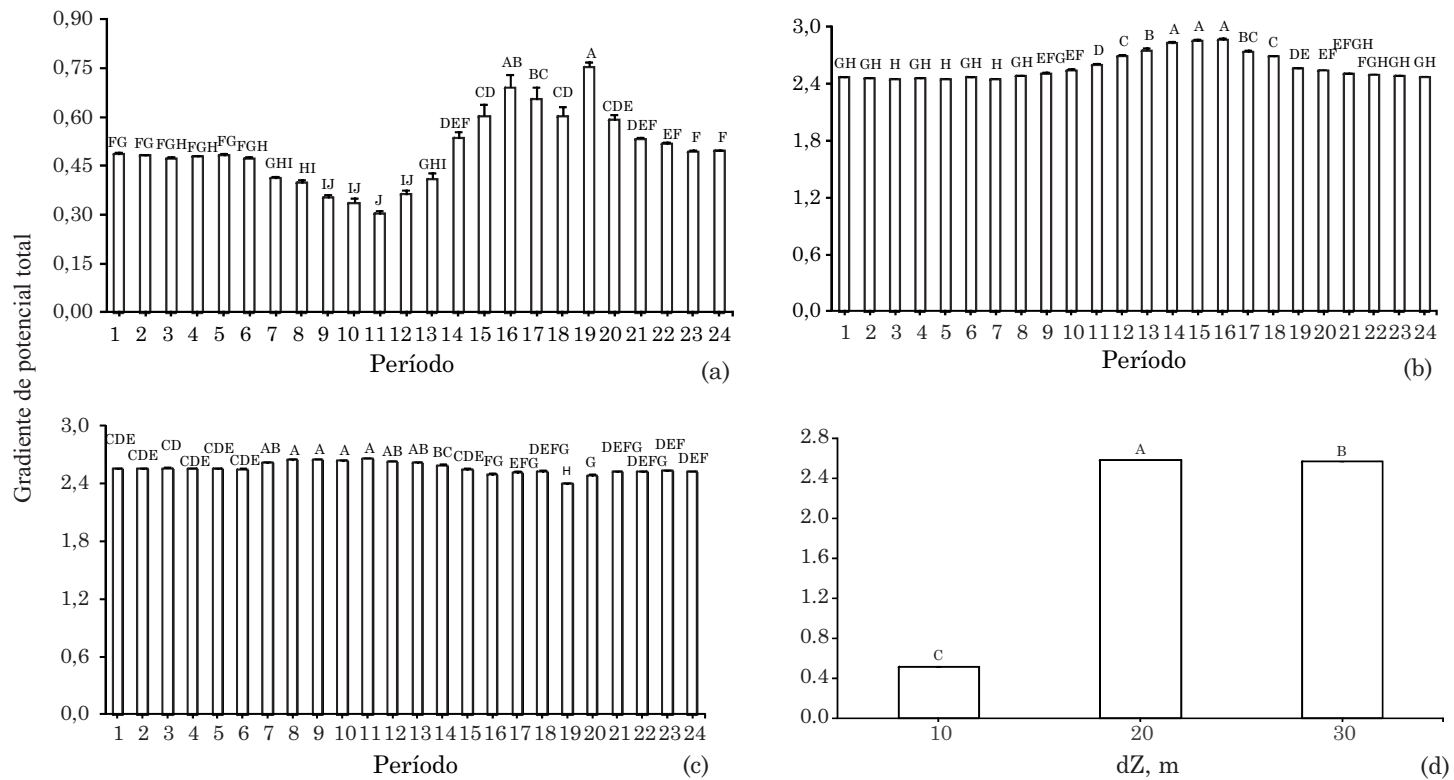

Figura 3. Gradiente de potencial total da água no solo nas profundidades de $0,25 \mathrm{~m}(\mathrm{a}, \mathrm{b}, \mathrm{c})$, calculado com $\Delta z$ de 0,10 m (tensiômetros nas profundidades de 0,20 e 0,30 m); 0,20 m (tensiômetros nas profundidades de 0,15 e $0,35 \mathrm{~m}$ ); e 0,30 m (tensiômetros nas profundidades de 0,10 e $0,40 \mathrm{~m}$ ), em função dos 24 períodos do dia e (d) comparação de médias pelo teste de Tukey a $5 \%$. 
de água mais elevado nas camadas superficiais do solo (Figura 1a), durante a redistribuição da água no solo, diminuiu o efeito da temperatura (tampão térmico) na medida do potencial mátrico feita no tensiômetro instalado a $0,15 \mathrm{~m}$ de profundidade, como pode ser observado pelo afastamento das curvas de potencial total da água no solo (Figura 2b), principalmente no período das 9 às $20 \mathrm{~h}$, quando as temperaturas foram mais elevadas. É importante observar também que o gradiente de potencial total calculado para o final do dia retornou para valores semelhantes aos das primeiras horas, reforçando a influência da temperatura na medida desse parâmetro.

\section{CONCLUSÕES}

1. O potencial mátrico e o gradiente de potencial total da água no solo sofreram influência da variação da temperatura atmosférica e do solo, ocasionando diminuição de ambos nas horas mais quentes do dia.

2. A recomendação é que as leituras dos tensiômetros sejam feitas nas primeiras sete horas do dia ou após as $18 \mathrm{~h}$. Para experimentos que necessitem de leituras ao longo do dia, como é o caso da fase inicial da determinação da condutividade hidráulica do solo, é indispensável proteger o sistema de medida da radiação solar direta.

\section{LITERATURA CITADA}

AZZOZ, R.H. \& ARSHAD, M.A. Laboratory calibration of pressure transducer-tensiometer system for hydraulic studies. Can. J. Soil Sci., 74:315-319, 1994.

BAKKER, G.; van der PLOEG, M.J.; ROOIJ, G.H.; HOOGENDAM, C.W.; GOOREN, H.P.A.; HUISKES, C.; KOOPAL, L.K. \& KRUIDHOF, H. New polymer tensiometers: Measuring matric pressure down to the wilting point. Vadose Zone J., 6:196-202, 2007.

BRITO, A.S.; LIBARDI, P.L.; MOTA, J.C.A. \& MORAES, S.O. Desempenho do tensiômetro com diferentes sistemas de leitura. R. Bras. Ci. Solo, 33:17-24, 2009.

BUCHTER, B.; HINS, C.; WYNDLER, H. \& FLUHLER, H. Evaluation of temperature and bypass flow sensitivity of tensiometers in a field soil. Geoderma, 87:281-291, 1999.

BUTTERS, G.L. \& CARDON, G.E. Temperature effects on air-pocket tensiometers. Soil Sci., 163:677-685, 1998.
COELHO, S.L. \& TEIXEIRA, A.S. Avaliação do tensiômetro eletrônico no monitoramento do potencial matricial da água no solo. Eng. Agríc., 24:536-545, 2004.

DEXTER, A.R.; RICHARD, G.; CZYZ, E.A. \& GIOT, G. Changes in the matric potential of soil water with time and temperature. Soil Sci., 175:320-328, 2010.

HAISE, H.R. \& KELLEY, O.J. Causes of diurnal fluctuations of tensiometers. Soil Sci., 70:301-313, 1950.

HUBBELL, J.M. \& SISSON, J.B. Advanced tensiometer for shallow or deep soil water potential measurements. Soil Sci., 163:271-277, 1998.

KLEIN, V.A. Uma proposta de irrigação automática controlada por tensiômetros. R. Bras. Agroci., 7:231-234, 2001.

KLEIN, V.A.; CÂMARA, R.K.; SIMON, M.A. \& BIASUZ JÚNIOR, I.J. Avaliação de sensores de resistência elétrica e tensiômetros com transdutores de tensão para determinação do potencial de água do solo. R. Ci. Agrovet., 3:80-86, 2004.

MARTHALER, H.P.; VOGELSANGER, W.; RICHARD, F. \& WIERENGA, P.J. A pressure transducer for field tensiometers. Soil Sci. Soc. Am. J., 47:624-627, 1983.

MORAES, N.B.; MEDEIROS, J.F.; LEVIEN, S.L.A. \& OLIVEIRA, A.M.S. Avaliação de cápsulas de cerâmica de instrumentos de medida de tensão usados em tensiômetros. R. Bras. Eng. Agríc. Amb., 10:58-63, 2006.

NYRAN, J.W. \& DRENNON, B.J. Tensiometer data acquisition system for hydrologic studies requiring high temporal resolution. Soil Sci. Soc. Am. J., 54:293-296, 1990.

OR, D. Who invented the tensiometer? Soil Sci. Soc. Am. J., $65: 1-3,2001$

van der PLOEG, M.J.; GOOREN, H.P.A.; BAKKER, G. \& ROOIJ, G.H. Matric potential measurements by polymer tensiometers in cropped lysimeters under water-stressed conditions. Vadose Zone J., 7:1048-1054, 2008.

PACHECO, F.B.P.; REICHARDT, K. \& TUON, R.L. Variabilidade espacial e temporal do potencial mátrico da água em Terra Roxa Estrutura. Sci. Agríc., 51:327-334, 1994.

WANG, F.; KANG, Y.; LIU, S. \& HOU, X. Effects of soil matric potential on potato growth under drip irrigation in the North China Plain. Agric. Water Manage., 88:34-42, 2007.

WARRICK, A.W.; WIERENGA, P.J.; YOUNG, M.H. \& MUSIL, S.A. Diurnal fluctuations of tensiometric readings due to surface temperature changes. Water Resour. Res., $34: 2863-2869,1998$

WHALLEY, W. R. ; CLARK, L. J.; TAKE, W. A.; BIRD, N. R. A.; LEECH, P. K.; COPE, R. E.; WATTS, C. W. A porousmatrix sensor to measure the matric potential of soil water in the field. Eur. J. Soil Sci., 58:18-25, 2007. 